\title{
I-WARP: Individual Water mAin Renewal Planner
}

\author{
Y. Kleiner and B. Rajani \\ National Research Council Canada, Ottawa, Ontario, Canada
}

Received: 9 December 2009 - Published in Drink. Water Eng. Sci. Discuss.: 13 January 2010

Revised: 6 May 2010 - Accepted: 7 May 2010 - Published: 18 May 2010

\begin{abstract}
I-WARP is based upon a nonhomogeneous Poisson approach to model breakage rates in individual water mains. The structural deterioration of water mains and their subsequent failure are affected by many factors, both static (e.g., pipe material, pipe size, age (vintage), soil type) and dynamic (e.g., climate, cathodic protection, pressure zone changes). I-WARP allows for the consideration of both static and dynamic factors in the statistical analysis of historical breakage patterns. This paper describes the mathematical approach and demonstrates its application with the help of a case study. The research project within which I-WARP was developed, was jointly funded by the National Research Council of Canada (NRC), and the Water Research foundation (formerly known as the American Water Works Association Research Foundation - AwwaRF) and supported by water utilities from USA and Canada.
\end{abstract}

\section{Introduction}

The use of statistical methods to discern patterns of historical breakage rates and use them to predict water main breaks has been widely documented. Kleiner and Rajani (2001) provided a comprehensive review of approaches and methods that had been developed prior to their review. Since then, several more methods have been proposed, such as those by Park and Loganathan (2002), Mailhot et al. (2003), Dridi et al. (2005), Giustolisi et al. (2005), Watson et al. (2006), Giustolisi and Berardi (2007), Boxall et al. (2007), Le Gat (Le Gat, Y.: Extending the Yule Process to model recurrent failures of pressure pipes, private communication, 2008.) and Economou et al. (2008) to name but a few.

Many factors, operational, environmental and pipeintrinsic factors, jointly affect the breakage rate of a water main. While not all pipes are created equal (even pipes of the same material and size), it is normally assumed that pipes that share a specific intrinsic property, such as material, or diameter, can be expected to have the same breakage pattern, all else being equal. However, non-pipe-intrinsic factors may have varying effect on the breakage patterns of different pipes, even if all else is equal. For example, two pipes of the same material, diameter, age, etc. can be impacted differently by climate. These differences are due to variability for which we may never have enough data to account. At the same time, it is unreasonable to perform a statistical analysis on the breakage pattern of a single pipe because there often are insufficient breaks to conduct a credible analysis. For this reason, the forecasting of breaks in individual water mains has proven to be quite a challenge.

In this paper we present I-WARP (Individual Water mAin Renewal Planner), which is a tool to analyse the historical breakage patterns of individual water mains. I-WARP is based on the assumption that breaks on an individual pipe occur as a non-homogeneous Poisson process (NHPP). NHPP has been suggested by others to model the same phenomenon (e.g., Constantine and Darroch, 1993; Røstum, 2000; Jarrett et al., 2003, among others). The approach proposed here differs from others in that allows for the consideration of dynamic factors, while existing NHPP approaches consider only static factors (i.e., pipe-intrinsic).

The rest of this paper is organised as follows: Sect. 2 provides the theoretical background for the model, Sect. 3 discusses issues related to the use of specific covariates, Sect. 4 describes the Zero-Inflated Poisson concept, which is provided as an option in I-WARP, Sect. 5 provides details on the testing and validation of I-WARP results, Sect. 6 provides a case study to illustrate the model application and Sect. 7 provides summary and conclusions. 


\section{Non-homogeneous Poisson-based model}

In the proposed model we assume that breaks at year $t$ for an individual pipe $i$ are Poisson arrivals with mean intensity $\lambda_{i, t}$. Therefore, the probability of observing $k_{i, t}$ breaks is given by

$P\left(k_{i, t}\right)=\frac{\lambda_{i, t}^{k_{i, t}} \cdot \exp \left(-\lambda_{i, t}\right)}{k_{i, t} !}$

$\lambda_{i, t}=\exp \left[\alpha_{o}+\theta \tau\left(g_{i, t}\right)+\boldsymbol{\alpha} \boldsymbol{z}^{i}+\boldsymbol{\beta} \boldsymbol{p}^{t}+\boldsymbol{\gamma} \boldsymbol{q}^{i, t}\right]$

where $\alpha_{o}$ is a constant, $\tau\left(g_{i, t}\right)$ is the age covariate, and $\theta$ is its coefficient, $g_{i, t}$ is the age of pipe $i$ at year $t ; z^{i}$ is a row vector of pipe-dependent covariates (e.g., length, diameter, etc.) and $\boldsymbol{\alpha}$ is a column vector of the corresponding coefficients; $\boldsymbol{p}^{t}$ is a row vector of time-dependent covariates (e.g., climate) and $\boldsymbol{\beta}$ is a column vector of the corresponding coefficients; $\boldsymbol{q}^{i, t}$ is a row vector of both pipe-dependent and time-dependent covariates (e.g., number of known previous failure - NOKPF, cathodic protection) and $\gamma$ is a column vector of the corresponding coefficients. Note that if $\tau\left(g_{i, t}\right)=g_{i, t}$ then the aging is exponential, i.e., $\lambda$ is an exponential function of pipe age, whereas if $\tau(t)=\log _{e}\left(g_{i, t}\right)$ then $\lambda$ becomes a power function of pipe age. Year $t$ is taken relative to the first year for which breakage records are available. Coefficients are obtained using the maximum likelihood method.

\section{Covariates}

\subsection{Pipe-dependent}

Pipe-dependent covariates can be considered explicitly in the probabilistic model or implicitly by partitioning the data into homogeneous populations with respect to these covariates. The explicit consideration introduces some limitations. For example, if one includes pipe diameter in the $z^{i}$ vector of covariates the mean breakage intensities for all pipes with the same diameter are assumed to be impacted by the same magnitude. Moreover, this inclusion implies that pipes of different diameters are impacted proportionally to the exponent of the difference between their respective diameters. For example, if pipe diameter is a covariate with a negative coefficient $-\alpha$ then the impact on breakage intensity of a $200 \mathrm{~mm}$ pipediameter is smaller than that of a $100 \mathrm{~mm}$ diameter pipe by $\exp [-\alpha(200-100)]$, and so forth. Alternatively, one can partition the population of pipes into groups, each comprising only pipes with the same diameter. Each group is then analysed separately, producing group specific coefficients. The latter approach encompasses two advantages: (a) removal of the forced proportionality described above, and (b) relaxation of the implied assumption that each covariate affects the mean intensity independently. These two advantages come at the cost of reduced statistical significance due to analysis of smaller pipe populations (groups).

The model represented in equation (1) is general enough to accommodate both approaches, namely explicit consideration of pipe-dependent covariates and consideration by grouping. I-WARP in its current form is implemented with the second approach; hence it is demonstrated here with homogeneous groups. It should be noted, however that it would take little effort to implement I-WARP with the first approach.

I-WARP uses pipe diameter as a grouping criterion, as well as categorical properties such as pipe material, soil type, service connections or any such property that may be supported by available data. For example, if a utility has asbestos cement (AC) and cast iron (CI) pipes, with diameters of $6^{\prime \prime}$ and $8^{\prime \prime}$, ideally, 4 homogeneous groups could be formed (AC$6^{\prime \prime}, \mathrm{AC}-8^{\prime \prime}, \mathrm{CI}-6^{\prime \prime}$, and CI-8") and I-WARP applied to each group separately. In situations where too few data exist for fine grouping, groups could be combined for analysis (some engineering judgment would be useful for deciding on which groups to combine). Readers should note that in situations where too few data exist for one or more classes of inventory the accuracy of the results will likely be compromised regardless of the type of model or whether or not covariates are considered explicitly or implicitly through grouping.

Pipe length and pipe cluster are always used as explicit covariates. Pipe cluster is a surrogate for spatial covariates for which data may not always be available. Water utilities often lack data that are (directly or indirectly) geographically related, such as soil data, overburden characteristics (land development, traffic loading), historical installation practices, groundwater fluctuations, transient pressures, poor bedding, etc. These data, if available, may sometimes help to "explain" variations in breakage rates among individual water mains. In the absence of such data, the proximity of a pipe to a cluster of historical breaks may serve as a useful surrogate. The details on how to form pipe clusters and the cluster covariates are not discussed in this paper.

\subsection{Time-dependent}

In the category of time-dependent covariates, three climaterelated covariates were considered, namely freezing index (FI), cumulative rain deficit (RDc) and snapshot rain deficit (RDs). Kleiner and Rajani (2004) provided a detailed introduction and a rational for using these covariates. FI is a surrogate for the severity of a winter, $\mathrm{RDc}$ is a surrogate for average annual soil moisture and RDs is a surrogate for locked-in winter soil moisture (appropriate for cold regions, where soil can freeze in the winter).

I-WARP is not restricted in the way it can consider timedependent covariates. In fact any phenomenon, suspected as a contributor to observed variations in breakage rate, can be considered in the model, provided there exists a time series describing this phenomenon over the observed period of time. Such phenomena can be represented quantitatively or qualitatively. For example, in one of the case studies documented in this research, uncharacteristically elevated breakage rates were observed in a network during two noncontiguous years. A quick inquiry revealed that the network 
experienced pump station failures in those years, which resulted in high breakage rates probably due to transient pressures. A qualitative time series describing this phenomenon was incorporated in the model and the calibration results improved significantly. Other phenomena represented by timeseries could include pressure regime changes overtime, leak detection campaigns, changes over time of overburden (traffic) intensity, etc.

\subsection{Pipe and time-dependent}

In its current form I-WARP considers three pipe-dependent and time-dependent covariates, namely, number of known previous failures (NOKPF), a covariate related to hot spot cathodic protection (HSCP) and a covariate related to retrofit cathodic protection (RetroCP). To ensure stability in the maximum likelihood calculations it may be beneficial to use the $\log _{e}$ of NOKPF as the covariate, especially when there are substantial discrepancies between breakage rates of individual pipes in the group.

The dependency of pipe failure rate on the number of previous failures has been observed by others (e.g., Andreou et al., 1987; Rostum, 2000). Typically, covariates used were break order, or number of breaks observed since installation. As the vast majority of water utilities do not have a complete breakage history of pipes since installation (left truncated data), we selected a more realistically available (if less rigorous) covariate of previously known number of failures. The aforementioned cathodic protection covariates are not described in this paper

\subsection{Data requirements}

It is clear that rich and accurate data increase the quality and credibility of the results. The authors' experience in the field shows that no water utility has a perfect dataset and there are huge differences in data quality between water utilities. Even advanced water utilities with sophisticated GIS can have severe deficiencies in the quality of data that pertain sometimes to pipes laid more than 100 years ago. Data cleansing is always encouraged and the I-WARP program application has some (limited) built-in tools to filter out inconsistencies.

The authors' sense is that it is prudent to have at least 5 years worth of failure data in order to obtain meaningful results, but of course a longer dataset is expected to increase accuracy and significance of results. Pipe-dependent data (material, diameter, vintage, service connections, etc.) are used as grouping criteria and the more data are available the more refined the grouping, likely yielding more refined and accurate results.

\section{The Zero-Inflated Poisson (ZIP) process}

In reality, most water mains fail relatively rarely, which means that in a typical data set many (if not most) of our data points will have the observed value $k_{i, t}=0$ (i.e., zero breaks observed for pipe $i$ at year $t$ - see Eq. 1). It has been observed (e.g., Lambert, 1992) that a counting process with many zeros (i.e., many more than what is expected from Equation 1) cannot be adequately represented by a Poisson process. Lambert (1992) proposed a technique she called "Zero-Inflated Poisson" (ZIP) regression, for handling zero inflated count data. In this approach, the counting process at hand is produced simultaneously by two mechanisms, namely a zero generating process and a Poisson process. Economou et al. (2008) used this approach in their model to predict pipe breakage rates, and called the probability of obtaining a zero data point "the natural tendency of the pipe to resist failure". I-WARP allows the option of incorporating the ZIP process in the analysis, as it can (but is not guaranteed to) improve prediction accuracy. When ZIP is considered the probability of observing $k_{i, t}$ breaks (at year $t$ for an individual pipe $i$ ) becomes

$$
\begin{gathered}
P\left(k_{i, t}\right)= \begin{cases}G_{i, t}+\left(1-G_{i, t}\right) e^{-\lambda_{i, t}} & \text { for } k_{i, t}=0 \\
\left(1-G_{i, t}\right) \lambda_{i, t}^{k_{i, t}} e^{-\lambda_{i, t}} / k_{i, t} ! & \text { for } k_{i, t}>0\end{cases} \\
i=1,2, \ldots, N ; t=1,2, \ldots, T
\end{gathered}
$$

where $N$ is the number of pipes and $T$ is the number of years of available breakage data, $G_{i, t}$ is the parameter of the second mechanism (the first in the Poisson process) that produces $k_{i, t}=0$ with probability $G_{i, t}$. It is convenient to formulate $G_{i, t}$ in a logit form because its value must lie in the interval $[0$, 1], i.e.,

$\operatorname{Logit}\left(G_{i, t}\right)=f$ (some covariates)

or

$G_{i, t}=\frac{e^{f(\cdot)}}{1+e^{f(\cdot)}}$

It is reasonable to assume that $G_{i, t}$ is generally influenced by the same covariates that influence the mean intensity $\lambda_{i, t}$. Therefore we define $G_{i, t}$ as a function of $\lambda_{i, t}$

$G_{i, t}=\frac{e^{g_{0}-\lambda_{i, t}}}{1+e^{g_{0}-\lambda_{i, t}}}$

where $g_{o}$ is the ZIP coefficient. Note that with this formulation $G_{i, t}$ tends to zero as $\lambda_{i, t}$ increases and $G_{i, t}$ tends to unity as $\lambda_{i, t}$ decreases.

\section{Testing and validating I-WARP}

The testing protocol consists of three steps: (a) training the model (discern coefficients) on data of $T$ years (training period), (b) use the discerned coefficients to forecast breaks in subsequent $V$ years (validation period), and (c) compare the forecasted and observed breaks in the validation period.

The evaluation of how well the trained model fits observed data (step (a)) is challenging for this type of model because observed data are integers (counts of breaks) while 
the model predicts expected number of breaks (referred to earlier as "mean intensity" or "mean rate of occurrence" of failure), which are real numbers. Therefore, measures like root mean square deviations or generalized $R^{2}$ (Nagelkerke, 1991) are not well suited for this type of model. Consequently, two goodness of fit measures were used, namely a pipe-dimension coefficient of determination, $p R^{2}$, and a time-dimension coefficient of determination, $t R^{2}$.

$$
\begin{array}{r}
p R^{2}=1-\frac{\sum_{i=1}^{N}\left(\sum_{t=1}^{T} k_{i, t}-\sum_{t=1}^{T} \hat{\lambda}_{i, t}\right)^{2}}{\sum_{i=1}^{N}\left(\sum_{t=1}^{T} k_{i, t}-\frac{1}{N} \sum_{t=1}^{T} \sum_{i=1}^{N} k_{i, t}\right)^{2}} \\
t R^{2}=1-\frac{\sum_{t=1}^{T}\left(\sum_{i=1}^{N} k_{i, t}-\sum_{i=1}^{N} \hat{\lambda}_{i, t}\right)^{2}}{\sum_{t=1}^{T}\left(\sum_{i=1}^{N} k_{i, t}-\frac{1}{T} \sum_{t=1}^{T} \sum_{i=1}^{N} k_{i, t}\right)^{2}}
\end{array}
$$

where $k_{i, t}$ is the observed - and $\hat{\lambda}_{i, t}$ is the estimated - number of breaks in pipe $i$ at year $t$. Essentially $p R^{2}$ computes the coefficient of determination between the observed and predicted data, where the data are aggregated by pipe (i.e., for each pipe it compares the total number of observed breaks with predicted values over training period $T$ ). Similarly, $t R^{2}$ computes the coefficient of determination between the observed and predicted data, where the data are aggregated by year (i.e., for each year in $T$ it compares the total number of observed breaks to predicted values in all $N$ pipes).

Equation (5) can also be used to evaluate results of step (c), i.e., the forecasting accuracy. In addition, we used a measure to assess the "ranking ability" (in terms of forecasted number of breaks) of the forecast. This measure is explained as follows: if in a group $P$, comprising $p$ pipes there is a subset $N$ comprising $n$ pipes that have at least $m$ breaks, if one draws at random $n$ pipes out of $P$, then $\mathrm{P}$-value is defined as the probability that at least $k$ pipes (from those drawn at random) are members of $N$. It can be shown that $k$ is a random variable with a hyper-geometric probability distribution, and P-value can thus be computed. For example, suppose that in a group of 100 pipes 5 pipes are observed to have experienced at 4 breaks (each) during the validation period. If 5 pipes are selected at random from the 100 pipes, the probability that at least two of those selected will have 5 breaks in the validation period is $\mathrm{P}$-value $\approx 0.019$. It follows that if the model succeeds in identifying 2 out of the 5 highest breaking pipes (in a group of 100 pipes) it is doing significantly better than a random draw (which has only about $2 \%$ chance to do as well).

The statistical significance of the contribution of each covariate to the model accuracy can be determined by e.g., the likelihood ratio test (e.g., Ansel and Phillips, 1994). This topic, however, is not discussed in this paper.

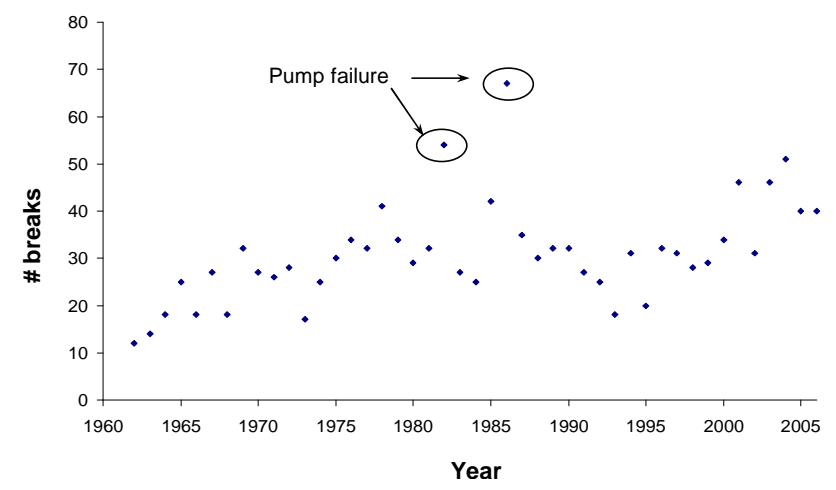

Figure 1. Breaks aggregated by year.

\section{Case study}

We use a data set obtained from a water utility in western Canada to illustrate the performance of I-WARP. The data set comprises 1091 individual pipes (each with a minimum length of $20 \mathrm{~m}$ ) with a total length of $146.6 \mathrm{~km}$, all $150 \mathrm{~mm}$ diameter unlined cast iron pipes, installed between 1956 and 1960. Available pipe data included pipe material, diameter, installation year, length, and $x-y$ coordinates of pipe nodes. Any intervention that involved pipe exposure and repair was considered a "break" event, for which date, type and related pipe ID was provided. Full year breakage data were available for the years 1961-2006. Some information on cathodic protection was also provided but is not used in this example. Climate data for the analysis years were obtained from Environment Canada. I-WARP was trained on 40 years failure data from 1962 to 2001 and the coefficients obtained from training were used to forecast breaks for validation for the subsequent 5 years, i.e., 2002-2006.

The temporal distribution of the breaks is illustrated in Fig. 1. Note the two outliers in 1982 and 1986. The utility engineering staff noted that pumping station failures occurred in these years with the consequence of a significant spike in the number of pipe failures. As discussed earlier, IWARP allows the inclusion of such information by means of a user-defined time-dependent covariate. Figure 2 illustrates the training and validation results with temporal aggregation (top) and pipe-aggregation (bottom). Table 1 provides the ranking ability of the model. Note that the ranking ability is for the validation (not training) period.

The ageing covariate $\tau(t)=\log _{e}\left(g_{i, t}\right)$ was used in this case study. An examination of the coefficients (Fig. 2) reveals that background ageing was therefore proportional to sixth root (power of about 0.16 ) of pipe age. The impact of climate covariates on the model was inconsistent. Freezing index (FI) showed little impact, rain deficit (RDc) appeared to have a more significant impact, but the impact of snapshot rain deficit (RDs) was in a counter intuitive direction (negative coefficient). Water mains of this water utility are 


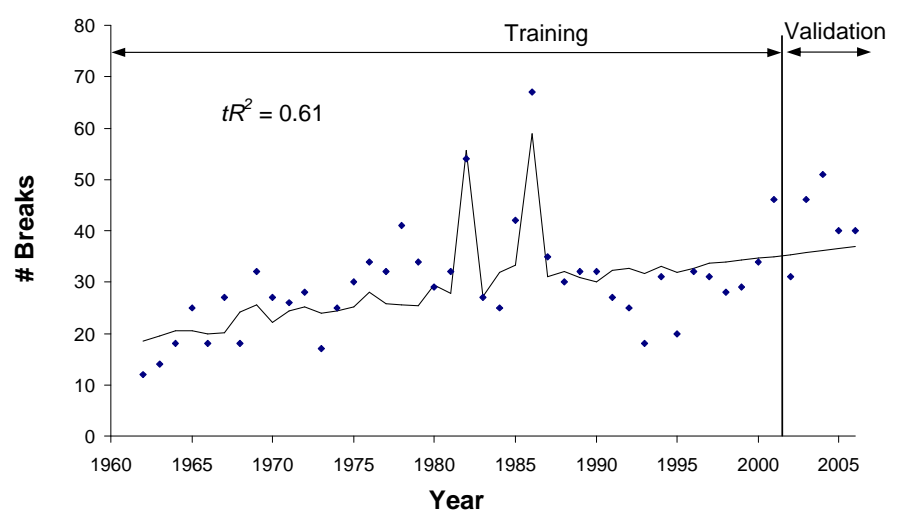

\begin{tabular}{lccccccccc}
\hline Covariate: & constant & Age & Length & NOKPF & FI & RDs & RDc & Pump failures & ZIP \\
\hline Coefficient & -6.182 & 0.16 & 0.69 & 0.44 & -0.01 & -0.12 & 0.13 & 0.66 & 1.22 \\
\hline
\end{tabular}
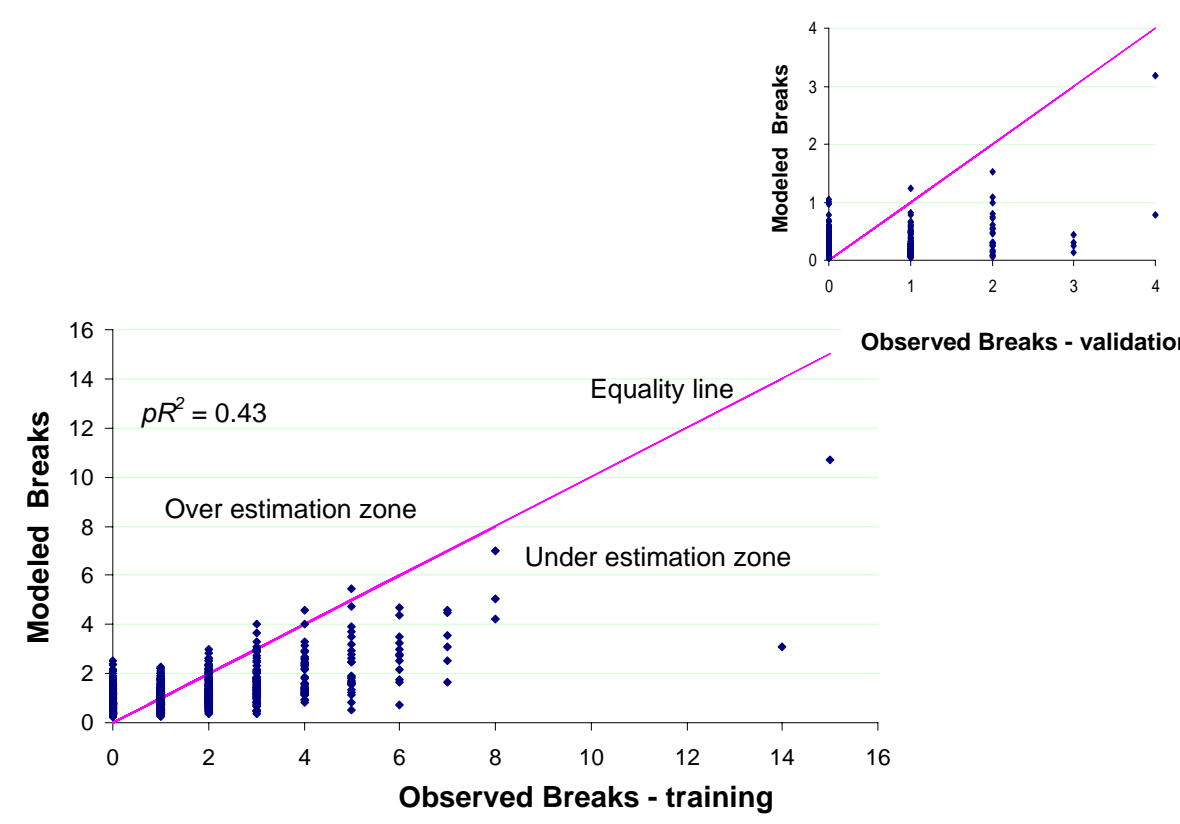

Figure 2. Training and validation results.

Table 1. Ranking ability of the model (validation period).

\begin{tabular}{lccccc}
\hline$N$ break(s) in the validation period & $n=1$ & $n=2$ & $n=3$ & $n=4$ & $n=5$ \\
\hline \# pipes with at least $n$ (observed) break(s) out of 1091 pipes in group & 170 & 30 & 6 & 2 & 0 \\
\# of pipes, $k$, identified correctly & 53 & 9 & 1 & 1 & N/A \\
P-value (probability of identifying $k$ pipes by pure chance) & 0.0 & 0.0 & 0.033 & 0.004 & \\
\hline
\end{tabular}

typically buried at a depth of $2.4 \mathrm{~m}$, which may explain the insignificant impact of FI, but not the negative sign of RDs. The positive sign of NOKPF may point to a "worse than old" condition (in repairable systems four repair-related conditions are observed, "good as new", "good as old", "better than old" and "worse than old"). The length covariate in this case study was taken as the $\log _{e}$ of pipe length, which means that the number of estimated break was proportional to the length of the pipe raised to the power of 0.7 , which is a relatively strong dependency. 
The results seem to indicate that in this case study:

- I-WARP tended to be quite accurate in predicting total numbers of breaks:

\begin{tabular}{lcc}
\hline & \multicolumn{2}{c}{ \# Breaks } \\
\hline & Training period & Validation period \\
\hline Observed & 1184 & 208 \\
Predicted & 1173 & 189
\end{tabular}

- I-WARP was rather successful in estimating the total number of breaks per year of the entire group $\left(t R^{2}=\right.$ $0.61)$

- I-WARP was not as successful in estimating the number of breaks per pipe $\left(p R^{2}=0.43\right)$. It tended to overestimate the number of breaks for pipes that experienced few breaks, while under-estimating the number of breaks for those pipes that experienced a higher number of breaks. A similar tendency has been observed by others, e.g., Rostum (2000). This may be due to the fact that there are many pipes with zero or few breaks and only a few pipes with many breaks.

- I-WARP displayed a statistically significant ranking ability in its forecast, which would help to prioritise pipes for renewal.

Additionally, when we varied the length of the validation period we have observed that longer validation periods resulted in improved ranking ability of the forecast. This may be because I-WARP forecasts mean intensities, while observed values are random events. The longer the forecast period the more these observed values would tend towards their means.

\section{Summary and conclusions}

I-WARP is a non-homogeneous Poisson process-based model, which considers three classes of covariates, pipedependent, time-dependent and pipe and time dependent. Some pipe-dependent covariates (e.g., pipe diameter, material, soil type, vintage, etc.) are considered implicitly through pipe grouping, while time-dependent (e.g., climate) and pipe and time dependent (e.g., NOKPF, cathodic protection) covariates are considered explicitly in the statistical analysis.

I-WARP was demonstrated using a case study. The model was trained on 40 years of historical breakage data and the trained model used to forecast breaks in the subsequent 5 years. While prediction of aggregate number of breaks per year was good, the aggregated total number of breaks per pipe was over estimated for pipes with few historical breaks and underestimated for pipes with many historical breaks. Ranking ability was statistically quite significant.

I-WARP is general enough to be used in many circumstances. Pipe-dependent covariates can be accommodated by simply creating a relevant homogeneous group. The underlying model is also flexible enough to consider any timedependent covariates as well, provided data are available to support such an analysis. In its current form I-WARP supports 3 climate related covariates (freezing index, rain deficitcumulative and rain-deficit-snapshot), two types of cathodic protection covariates and two user-defined time series. Time series representing time-dependent covariates can be accommodated if they are thought to have an impact on observed historical breakage patterns.

A prototype computer application was created for the application of I-WARP. It will soon be publicly available through WRF.

Acknowledgement. I-WARP was developed as part of a research project, which was co-sponsored by the Water Research Foundation (WRF - formerly known as the American water Works Association Research Foundation - AwwaRF), the NRC and water utilities from the United States and Canada. The authors wish to acknowledge the invaluable help provided by Ahmed Abdel Akher at the NRC.

Edited by: J. Vreeburg

\section{References}

Andreou, S. A., Marks, D. H., and Clark, R. M.: A new methodology for modeling break failure patterns in deteriorating water distribution systems: Theory, Adv. Water Resour., 10, 2-10, 1987.

Ansell, J. I. and Phillips, M.J.: Practical methods for reliability data analysis, Oxford University Press, Oxford, UK, 1994.

Boxall, J. B., O'Hagen, A., Pooladsaz, S., Saul, A., and Unwin, D.: Estimation of burst rate in water distribution mains, Proceedings of the Institution of Civil Engineers, Water Management I60, Issue WM2, 73-82, June 2007.

Constantine, A. G. and Darroch, J. N.: Pipeline reliability: stochastic models in engineering technology and management, edited by: Osaki, S. and Murthy, D. N. P., World Scientific Publishing Co., 1993.

Dridi, L., Mailhot, A., Parizeau, M., and Villeneuve, J. P.: A strategy for optimal replacement of water pipes integrating structural and hydraulic indicators based on a statistical water pipe break model, Proceedings of the 8th International Conference on Computing and Control for the Water Industry, Univ. of Exeter, UK, 65-70, September 2005.

Economou, T., Kapelan, Z., and Bailey, T.: A Zero-Inflated Bayesian Model for the Prediction of Water Pipe Bursts, Proc. 10th International Water Distribution System Analysis Conference, CD-ROM edition, Kruger National Park, South Africa, 2008.

Giustolisi, O., Laucelli, D., and Savic D. A.: A decision support framework fro short time planning of rehabilitation, Proceedings of Computer and Control in Water Industry (CCWI), 1, 39-44, 2005.

Giustolisi, O. and Berardi, L.: Pipe level burst prediction using EPR and MCS-EPR, Proceedings of the Combined International Conference of Computing and Control for the Water Industry (CCWI2007) and Sustainable Urban water Management (SUWM2007) on Water Management Challenges in Global Change, Leicester, UK, September, 39-46, 2007. 
Jarrett, R., Hussain, O., and Van der Touw, J.: Reliability assessment of water pipelines using limited data, OzWater, Perth, Australia, 2003.

Kleiner, Y. and Rajani, B.: Comprehensive review of structural deterioration of water mains: statistical models, Urban Water, 3, 131-150, 2001.

Kleiner, Y. and Rajani, B.: Quantifying effectiveness of cathodic protection in water mains: theory, J. Infrastruct. Syst., ASCE, 10(2), 43-51, 2004.

Lambert, D.: Zero-Inflated Poisson regression, with an application to defects in manufacturing, Technometrics, 34, 1, 1-14, 1992.

Mailhot, A., Paulin, A., and Villeneuve, J.-P.: Optimal replacement of water pipes, Water Resour. Res., 39(5), 1136-1150, 2003.
Nagelkerke, N.: A Note on a General Definition of the Coefficient of Determination, Biometrika, 78(3), 691-692, 1991.

Park, S. and Loganathan, G. V.: Optimal pipe replacement analysis with a new pipe break prediction model, Journal of the Korean Society of Water and Wastewater, 16/6, 710-716, 2002.

Røstum, J.: Statistical modelling of pipe failures in water networks, $\mathrm{PhD}$ thesis, Norwegian University of Science and Technology, Trondheim, Norway, 2000.

Watson, T. G., Christian, C. D., Mason, A. J., Smith, M. H., and Meyer, R.: Bayesian-based pipe failure model, J. Hydroinform., IWA publishing, 06.4, 259-264, 2004. 